\title{
A LEAST SQUARES PETROV-GALERKIN FINITE ELEMENT METHOD FOR THE STATIONARY NAVIER-STOKES EQUATIONS
}

\author{
TIAN-XIAO ZHOU AND MIN-FU FENG
}

\begin{abstract}
In this paper, a Galerkin/least squares-type finite element method is proposed and analyzed for the stationary Navier-Stokes equations. The method is consistent and stable for any combination of discrete velocity and pressure spaces (without requiring a Babuška-Brezzi stability condition). The existence, uniqueness and convergence (at optimal rate) of the discrete solution is proved in the case of sufficient viscosity (or small data).
\end{abstract}

\section{INTRODUCTION}

For mixed finite element methods solving the stationary Navier-Stokes equations, it is an important convergence stability condition that the Babuška-Brezzi inequality holds for the combination of finite element subspaces (see $[1,13])$. Recently, in an attempt to circumvent this constraint, the so-called CBB [6] or stabilized finite element methods [2-5] have been developed, motivated by SD (or SUPG) methods [7, 8]. In addition to works [3-6] on the Stokes problems, the paper [9] proposed and analyzed a stabilized SD method for time-dependent $\mathrm{N}-\mathrm{S}$ equations, and a stabilized, piecewise discontinuous vorticity-stream function formulation of mesh-dependent type for stationary N-S equations has been discussed in the paper [10] based on so-called homology families of generalized variational principles.

The present paper considers the stationary N-S equations in primitive variables. In this direction, L. Tobiska and G. Lube [12] proposed a penalty finite element method of streamline diffusion type. It is a stabilized method in which the finite element spaces of velocity and pressure are not required to satisfy the discrete B-B condition. But it is not consistent with the exact solution, owing to the addition of the penalty term $\alpha(\nabla p, \nabla q)$, and the optimal estimates of convergence rate cannot be achieved. In this paper, another stabilized finite element method is studied, which is different from the method in [11, 12]. It is an application of the Galerkin/least squares method [14] and its alternative [16] to nonlinear equations. Least squares forms of residuals are added to the Galerkin method for enhancing its stability without degrading accuracy.

For the following presentation we introduce the following notation: $X=$ $V \times Q, V=H_{0}^{1}(\Omega)^{n}, Q=L_{0}^{2}(\Omega)=\left\{q \in L^{2}(\Omega) \mid \int_{\Omega} q d x=0\right\},(\circ, \circ)_{G}$ the

Received by the editor January 9, 1991 and, in revised form, August 21, 1991 and March 31, 1992.

1991 Mathematics Subject Classification. Primary 65N30.

The research was supported by the Chinese Aeronautical Science Foundation. 
inner product in $L^{2}(G)$ and $L^{2}(G)^{n}$, respectively, $G \subseteq \Omega$. Let $\|w\|_{k, p, G}$ and $|w|_{k, p, G}$ be the usual norm and seminorm on the Sobolev space $W^{k, p}(G)$, respectively. For vector-valued functions $u=\left(u_{1}, \ldots, u_{n}\right) \in W^{k, p}(G)^{n}$ and $v=\left(v_{1}, \ldots, v_{n}\right) \in L^{\infty}(G)^{n}$ we use the following norms and seminorms, respectively: $\|u\|_{k, p, G}^{p}=\sum_{i=1}^{n}\left\|u_{i}\right\|_{k, p, G}^{p}, \quad|u|_{k, p, G}^{p}=\sum_{i=1}^{n}\left|u_{i}\right|_{k, p, G}^{p},\|v\|_{0, \infty, G}=$ $\max _{i}\left\|v_{i}\right\|_{0, \infty, G}$. In the case of $G=\Omega$ and $p=2$ we omit the indices $G$ and $p$.

Throughout the paper, $C$ indicates a positive constant, possibly different at different occurrences, which is independent of the mesh parameter $h$, but may depend on $\Omega$, on the Reynolds number and other parameters introduced in this paper. Notations not especially explained are used with their usual meanings.

An outline of the paper follows. In $\S 2$ we present the new finite element variational formulation for the N-S equations. The existence and uniqueness of the finite element solution is studied in $\S 3$. Its error analysis is performed in $\S 4$, and concluding remarks are made in $\S 5$.

\section{Finite ELEMENT FORMULATION}

Let $\Omega$ be a convex domain with boundary $\Gamma$ in $R^{n}(n=2,3)$. We consider the following stationary Navier-Stokes equations with boundary conditions:

$$
\begin{aligned}
& -\mu \Delta u+(u \nabla) u+\nabla p=f \text { in } \Omega, \\
& \operatorname{div} u=0 \text { in } \Omega \text {, } \\
& u=0 \text { on } \Gamma \text {, }
\end{aligned}
$$

where $u=\left(u_{1}, \ldots, u_{n}\right)$ is the velocity vector, $p$ the pressure, $f=\left(f_{1}, \ldots, f_{n}\right)$ the body force, and $\mu$ the constant inverse Reynolds number. Problem (2.1) is equivalent to the following variational problem:

Find $(u, p) \in X$ such that for all $(v, q) \in X$

$$
\mu a(u, v)+b(u ; u, v)-(p, \operatorname{div} v)+(q, \operatorname{div} u)=(f, v),
$$

where

$$
\begin{aligned}
a(u, v) & =\int_{\Omega} \nabla u \nabla v d x, \\
\tilde{b}(u ; v, w) & =\sum_{i, j=1}^{n} \int_{\Omega} u_{i} \partial v_{j} / \partial x_{i} w_{j} d x \quad \forall u, v, w \in V, \\
b(u ; v, w) & =\frac{1}{2}\{\tilde{b}(u ; v, w)-\tilde{b}(u ; w, v)\} \quad \forall u, v, w \in V .
\end{aligned}
$$

We define

$$
N=\operatorname{Sup}_{u, v, w \in V} \frac{b(u ; v, w)}{|u|_{1}|v|_{1}|w|_{1}}, \quad\|f\|_{*}=\operatorname{Sup}_{v \in V} \frac{(f, v)}{|v|_{1}} .
$$

Theorem 2.1 [1]. If $f \in H^{-1}(\Omega)^{n}$, then the problem (2.2) has a solution which, in addition, is unique provided that $\mu^{-2} N\|f\|_{*}<1$.

Let $\left\{\mathscr{F}_{h}\right\}$ be a family of triangulations of $\Omega$ into affinely equivalent finite elements $K$ with $\Omega=\bigcup_{K \in \mathscr{F}_{h}} K$, which is assumed to be regular in the usual sense, and let $h_{K}=\operatorname{diam} K$. We also assume that $h / h_{K}<C, \forall K \in \mathscr{F}_{h}$, $h=\max _{K} h_{K}$, so that we can use inverse inequalities. 
We introduce the following finite element spaces of velocity and pressure:

$$
\begin{aligned}
& V_{h}(\Omega)=\left\{v \in H_{0}^{1}(\Omega)^{n}:\left.v\right|_{K} \in P_{l}(x), \forall K \in \mathscr{F}_{h}\right\}, \\
& Q_{h}(\Omega)=\left\{q \in Q \cap H^{1}(\Omega):\left.q\right|_{K} \in P_{k}(x), \forall K \in \mathscr{F}_{h}\right\} .
\end{aligned}
$$

Here, $P_{l}(x), P_{k}(x)$ denote piecewise polynomials of degree $l$ and $k$, respectively. We let $X_{h}=V_{h} \times Q_{h}$.

Paper [12] proposed the following penalty finite element method of streamline diffusion type with the penalty term $\alpha(\nabla p, \nabla q)$ for (2.2):

Find $\hat{u}_{h}=\left(u_{h}, p_{h}\right) \in X_{h}$ such that for all $\hat{v} \in X_{h}$

$$
\begin{aligned}
\mu a\left(u_{h},\right. & v)+b\left(u_{h} ; u_{h}, v\right)-\left(p_{h}, \operatorname{div} v\right)+\left(q, \operatorname{div} u_{h}\right) \\
& +\sum_{K} \delta_{K}\left(-\mu \Delta u_{h}+\left(u_{h} \nabla\right) u_{h}+\nabla p_{h},\left(u_{h} \nabla\right) v+\nabla q\right)_{K} \\
& +\alpha\left(\nabla p_{h}, \nabla q\right) \\
= & (f, v)+\sum_{K} \delta_{K}\left(f,\left(u_{h} \nabla\right) v+\nabla q\right)_{K} .
\end{aligned}
$$

In order to establish existence, uniqueness, and convergence of the solution of (2.3), the parameter $\delta_{K}$ is required to satisfy the condition $0<\delta_{K}<C_{1} \mu^{-1} h^{2}$, where $C_{1}$ is a certain constant.

In this paper, we present the following Petrov-Galerkin finite element formulation for problem (2.1): Find $\hat{u}_{h}=\left(u_{h}, p_{h}\right) \in X_{h}$ such that for all $\hat{v} \in X_{h}$

$$
\begin{aligned}
\mu a\left(u_{h}, v\right) & +b\left(u_{h} ; u_{h}, v\right)-\left(p_{h}, \operatorname{div} v\right)+\left(q, \operatorname{div} u_{h}\right) \\
& +\sum_{k} \delta_{K}\left(-\mu \Delta u_{h}+\left(u_{h} \nabla\right) u_{h}+\nabla p_{h},-\mu \Delta v+\left(u_{h} \nabla\right) v+\nabla q\right)_{K} \\
= & (f, v)+\sum_{K} \delta_{K}\left(f,-\mu \Delta v+\left(u_{h} \nabla\right) v+\nabla q\right)_{K},
\end{aligned}
$$

where $\delta_{K}=\alpha h_{K}^{2}$, and $\alpha>0$ is arbitrary.

For $u \in V, u_{h} \in V_{h}, \hat{v}=(v, q), \hat{w}=(w, r) \in V \times\left(Q \cap H^{1}(\Omega)\right)$, we define

$$
\begin{aligned}
B_{\delta}\left(u, u_{h} ; \hat{v}, \hat{w}\right)= & \mu a(v, w)+b(u ; v, w)-(q, \operatorname{div} w)+(r, \operatorname{div} v) \\
& +\sum_{K} \delta_{K}\left(-\mu \Delta v+u \nabla v+\nabla q,-\mu \Delta w+u_{h} \nabla w+\nabla r\right)_{K}, \\
L_{\delta}\left(u_{h} ; \hat{w}\right)= & (f, w)+\sum_{K} \delta_{K}\left(f,-\mu \Delta w+u_{h} \nabla w+\nabla r\right)_{K},
\end{aligned}
$$

where $\delta$ is the piecewise constant function defined by $\left.\delta\right|_{K}=\delta_{K}$. Then (2.4) can be rewritten in the following form: Find $\hat{u}_{h}=\left(u_{h}, p_{h}\right) \in X_{h}$ such that

$$
B_{\delta}\left(u_{h}, u_{h} ; \hat{u}_{h}, \hat{w}\right)=L_{\delta}\left(u_{h} ; \hat{w}\right) \quad \forall \hat{w} \in X_{h} .
$$

Remark 1. Assume $f$ belongs to $L^{2}(\Omega)^{n}$ and the solution $(u, p)$ of $(2.1)$ belongs to $\left(V \cap H^{2}(\Omega)^{n}\right) \times\left(Q \cap H^{1}(\Omega)\right)$; i.e., there holds

$$
-\mu \Delta u+u \nabla u+\nabla p=f \quad \text { in } L^{2}(\Omega)^{n} .
$$

Then $\hat{u}=(u, p)$ satisfies

$$
B_{\delta}\left(u, u_{h} ; \hat{u}, \hat{w}\right)=L_{\delta}\left(u_{h} ; \hat{w}\right) \quad \forall u_{h} \in V_{h}, \hat{w} \in X_{h},
$$

i.e., (2.5) is consistent with the exact solution of problem (2.1) or (2.2). 


\section{EXISTENCE AND UNIQUENESS OF THE FINITE ELEMENT SOLUTION}

For the discrete problem (2.5) we will establish existence and uniqueness of an approximate solution without requiring the B-B condition.

Theorem 3.1. If $f \in L^{2}(\Omega)^{n}$, then (2.5) has at least one solution $\hat{u}_{h}=\left(u_{h}, p_{h}\right)$ $\in X_{h}$.

Proof. We use Brouwer's fixed point theorem to prove our theorem. The proof proceeds in two steps.

(I) For a given $\hat{v}_{h} \in X_{h}$, the following linearized problem of (2.5) has a unique solution:

Find $\hat{u}_{h}=\left(u_{h}, p_{h}\right) \in X_{h}$ such that

$$
B_{\delta}\left(v_{h}, v_{h} ; \hat{u}_{h}, \hat{w}\right)=L_{\delta}\left(v_{h} ; \hat{w}\right) \quad \forall \hat{w} \in X_{h} .
$$

In fact, we have

$$
B_{\delta}\left(v_{h}, v_{h} ; \hat{u}_{h}, \hat{u}_{h}\right)=\mu\left|u_{h}\right|_{1}^{2}+\left\|\delta^{1 / 2}\left(-\mu \Delta u_{h}+v_{h} \nabla u_{h}+\nabla p_{h}\right)\right\|_{0, h}^{2},
$$

where $\|\circ\|_{0, h}=\left(\sum_{K}\|\circ\|_{0, K}^{2}\right)^{1 / 2}$. By virtue of the coercivity of the bilinear form $B_{\delta}\left(v_{h}, v_{h} ; \hat{u}, \hat{w}\right)$ there exists a unique solution of (3.1), and since

$$
\begin{aligned}
L_{\delta}\left(v_{h} ; \hat{u}_{h}\right)= & \left(f, u_{h}\right)+\sum_{K} \delta_{K}\left(f,-\mu \Delta u_{h}+v_{h} \nabla u_{h}+\nabla p_{h}\right)_{K} \\
\leq & \left(\mu^{-1}\|f\|_{*}^{2}+\left\|\delta^{1 / 2} f\right\|_{0}^{2}\right)^{1 / 2} \\
& \times\left(\mu\left|u_{h}\right|_{1}^{2}+\left\|\delta^{1 / 2}\left(-\mu \Delta u_{h}+v_{h} \nabla u_{h}+\nabla p_{h}\right)\right\|_{0, h}^{2}\right)^{1 / 2},
\end{aligned}
$$

we get

$$
\begin{aligned}
& \left(\mu\left|u_{h}\right|_{1}^{2}+\left\|\delta^{1 / 2}\left(-\mu \Delta u_{h}+v_{h} \nabla u_{h}+\nabla p_{h}\right)\right\|_{0, h}^{2}\right)^{1 / 2} \\
& \leq\left(\mu^{-1}\|f\|_{*}^{2}+\left\|\delta^{1 / 2} f\right\|_{0}^{2}\right)^{1 / 2}
\end{aligned}
$$

or

$$
\begin{aligned}
& \left(\left|u_{h}\right|_{1}^{2}+\mu^{-1}\left\|\delta^{1 / 2}\left(-\mu \Delta u_{h}+v_{h} \nabla u_{h}+\nabla p_{h}\right)\right\|_{0, h}^{2}\right)^{1 / 2} \\
& \leq \frac{1}{\mu}\left(\|f\|_{*}^{2}+\mu\left\|\delta^{1 / 2} f\right\|_{0}^{2}\right)^{1 / 2} .
\end{aligned}
$$

Therefore, for arbitrarily given $\hat{v}_{h} \in X_{h}$, the solution of (3.1) determines a map $F: \hat{v}_{h} \rightarrow \hat{u}_{h}=F\left(\hat{v}_{h}\right)$.

For convenience, let $1=\left(\|f\|_{*}^{2}+\mu\left\|\delta^{1 / 2} f\right\|_{0}^{2}\right)^{1 / 2}, R=1 / \mu$.

(II) For the set $B_{R}=\left\{\hat{v}_{h} \in X_{h}:\left|v_{h}\right|_{1} \leq R\right\}, F$ is a continuous map from $B_{R}$ to $B_{R}$. In fact, by virtue of the estimate (3.2), it is easy to show that $F: B_{R} \rightarrow B_{R}$. Thus, we only need to prove $F$ is continuous.

Let for arbitrarily given $\hat{v}_{h}^{i} \in B_{R}$ the approximations $\hat{u}_{h}^{i}=F\left(\hat{v}_{h}^{i}\right) \quad(i=1,2)$ be defined by (3.1); then we have

$$
B_{\delta}\left(v_{h}^{i}, v_{h}^{i} ; \hat{u}_{h}^{i}, \hat{w}\right)=L_{\delta}\left(v_{h}^{i} ; \hat{w}\right) \quad \forall \hat{w} \in X_{h}
$$

and

$$
\left(\left|u_{h}^{i}\right|_{1}^{2}+\mu^{-1}\left\|\delta^{1 / 2}\left(-\mu \Delta u_{h}^{i}+v_{h}^{i} \cdot \nabla u_{h}^{i}+\nabla p_{h}^{i}\right)\right\|_{0, h}^{2}\right)^{1 / 2} \leq R \quad(i=1,2) .
$$


By (3.3), we have

$$
\begin{aligned}
& B_{\delta}\left(v_{h}^{1}, v_{h}^{1}, \hat{u}_{h}^{1}, \hat{w}\right)-B_{\delta}\left(v_{h}^{2}, v_{h}^{2} ; \hat{u}_{h}^{2}, \hat{w}\right) \\
& \quad=\sum_{K} \delta_{K}\left(f,\left(v_{h}^{1}-v_{h}^{2}\right) \nabla w\right)_{K} \quad \forall \hat{w} \in X_{h} .
\end{aligned}
$$

Now we choose $\hat{w}=\hat{u}_{h}^{1}-\hat{u}_{h}^{2}$, i.e., $\hat{w}=(w, r)=\left(u_{h}^{1}-u_{h}^{2}, p_{h}^{1}-p_{h}^{2}\right)$. We have

$$
B_{\delta}\left(v_{h}^{1}, v_{h}^{1} ; \hat{w}, \hat{w}\right)=\mu|w|_{1}^{2}+\left\|\delta^{1 / 2}\left(-\mu \Delta w+v_{h}^{1} \nabla w+\nabla r\right)\right\|_{0, h}^{2}
$$

and

$$
\begin{aligned}
B_{\delta}\left(v_{h}^{1},\right. & \left.v_{h}^{1} ; \hat{w}, \hat{w}\right)=B_{\delta}\left(v_{h}^{1}, v_{h}^{1} ; \hat{u}_{h}^{1}, \hat{w}\right)-B_{\delta}\left(v_{h}^{1}, v_{h}^{1} ; \hat{u}_{h}^{2}, \hat{w}\right) \\
= & B_{\delta}\left(v_{h}^{2}, v_{h}^{2} ; \hat{u}_{h}^{2}, \hat{w}\right)-B_{\delta}\left(v_{h}^{1}, v_{h}^{1} ; \hat{u}_{h}^{2}, \hat{w}\right) \\
& +\sum_{K} \delta_{K}\left(f,\left(v_{h}^{1}-v_{h}^{2}\right) \nabla w\right)_{K} \quad(\text { by } 3.5) \\
= & b\left(v_{h}^{2}-v_{h}^{1} ; u_{h}^{2}, w\right) \\
& +\sum_{K} \delta_{K}\left(\left(v_{h}^{2}-v_{h}^{1}\right) \nabla u_{h}^{2},-\mu \Delta w+v_{h}^{1} \nabla w+\nabla r\right)_{K} \\
& +\sum_{K} \delta_{K}\left(-\mu \Delta u_{h}^{2}+v_{h}^{2} \nabla u_{h}^{2}+\nabla p_{h}^{2},\left(v_{h}^{2}-v_{h}^{1}\right) \nabla w\right)_{K} \\
& +\sum_{K} \delta_{K}\left(f,\left(v_{h}^{1}-v_{h}^{2}\right) \nabla w\right)_{K} \\
= & : S_{1}+S_{2}+S_{3}+S_{4} .
\end{aligned}
$$

For $S_{4}$, it is easy to get

$$
\left|S_{4}\right| \leq\left\|v_{h}^{1}-v_{h}^{2}\right\|_{0, \infty}|w|_{1}\|\delta f\|_{0} .
$$

By means of Sobolev's embedding theorem and an inverse inequality, we can prove that

$$
\|v\|_{0, \infty} \leq C_{0} h^{-\chi}|v|_{1} \quad \forall v \in V_{h}
$$

with $\chi>0$ arbitrary in the case of $n=2$ and $\chi=1 / 2$ in the case of $n=3$. Thus, (3.8) yields

$$
\left|S_{4}\right| \leq C_{0} h^{-\chi}\|\delta f\|_{0}\left|v_{h}^{1}-v_{h}^{2}\right|_{1}|w|_{1} \leq C_{0} \mu^{1 / 2} \delta_{M}^{1 / 2} h^{-\chi} R\left|v_{h}^{1}-v_{h}^{2}\right|_{1}|w|_{1},
$$

where $\delta_{M}=\max _{x \in \Omega} \delta=\alpha h^{2}$. Similarly, by (3.4), (3.9) and the CauchySchwarz inequality, we get$$
\text { (3.12) }\left|S_{2}\right| \leq C_{0} R h^{-\chi} \delta_{M}^{1 / 2}\left|v_{h}^{2}-v_{h}^{1}\right|_{1}\left\|\delta^{1 / 2}\left(-\mu \Delta w+v_{h}^{1} \nabla w+\nabla r\right)\right\|_{0, h} \text {, }
$$$$
\text { (3.13) }\left|S_{3}\right| \leq C_{0} \mu^{1 / 2} \delta_{M}^{1 / 2} h^{-\chi} R\left|v_{h}^{2}-v_{h}^{1}\right|_{1}|w|_{1} \text {. }
$$

Combining (3.10), (3.11), and (3.13), we have

$$
\left|S_{1}\right|+\left|S_{3}\right|+\left|S_{4}\right| \leq\left(N R+2 C_{0} \mu^{1 / 2} \delta_{M}^{1 / 2} h^{-\chi} R\right)\left|v_{h}^{1}-v_{h}^{2}\right|_{1}|w|_{1},
$$

i.e.,

$$
\left|S_{1}\right|+\left|S_{3}\right|+\left|S_{4}\right| \leq M(R)\left|v_{h}^{1}-v_{h}^{2}\right|_{1}|w|_{1} .
$$


By (3.12), (3.14) and Cauchy's inequality, we obtain

$$
\sum_{i=1}^{4}\left|S_{i}\right| \leq L(R)\left|v_{h}^{1}-v_{h}^{2}\right|_{1}\left(\mu|w|_{1}^{2}+\left\|\delta^{1 / 2}\left(-\mu \Delta w+v_{h}^{1} \nabla w+\nabla r\right)\right\|_{0, h}^{2}\right)^{1 / 2}
$$

Then, by (3.6), (3.7), (3.15), we finally get

$$
\left(\mu|w|_{1}^{2}+\left\|\delta^{1 / 2}\left(-\mu \Delta w+v_{h}^{1} \nabla w+\nabla r\right)\right\|_{0, h}^{2}\right)^{1 / 2} \leq L(R)\left|v_{h}^{1}-v_{h}^{2}\right|_{1},
$$

where $M(R), L(R)$ are constants independent of $\hat{v}_{h}^{i}$ and $\hat{u}_{h}^{i}$. Noting that $\hat{w}=\hat{u}_{h}^{1}-\hat{u}_{h}^{2}$ and (3.16), we conclude that $F$ is a continuous map from $B_{R}$ to $B_{R}$. By Brouwer's fixed point theory, this implies that $F$ has at least one fixed point $\hat{u}_{h}=F\left(\hat{u}_{h}\right)$, i.e., the problem (2.5) has at least one solution $\hat{u}_{h}=$ $\left(u_{h}, p_{h}\right) \in X_{h}$.

Theorem 3.2. Assume that $f \in L^{2}(\Omega)^{n}, \mu^{-2} N\|f\|_{*}<1$. Then there is a constant $h_{0}>0$ such that for all $h \leq h_{0}$ the problem (2.5) is uniquely solvable, and the solution $\hat{u}_{h}=\left(u_{h}, p_{h}\right)$ satisfies the estimate

$$
\begin{aligned}
& \left(\mu\left|u_{h}\right|_{1}^{2}+\left\|\delta^{1 / 2}\left(-\mu \Delta u_{h}+u_{h} \nabla u_{h}+\nabla p_{h}\right)\right\|_{0, h}^{2}\right)^{1 / 2} \\
& \leq\left(\mu^{-1}\|f\|_{*}^{2}+\left\|\delta^{1 / 2} f\right\|_{0}^{2}\right)^{1 / 2} .
\end{aligned}
$$

Proof. From Theorem 3.1 we know that the problem (2.5) has at least one solution $\hat{u}_{h}=\left(u_{h}, p_{h}\right) \in X_{h}$ such that

$$
B_{\delta}\left(u_{h}, u_{h} ; \hat{u}_{h}, \hat{w}\right)=L_{\delta}\left(u_{h} ; \hat{w}\right) \quad \forall \hat{w} \in X_{h} .
$$

Setting $\hat{w}=\hat{u}_{h}$ in (3.18), we have

$$
\begin{gathered}
B_{\delta}\left(u_{h}, u_{h} ; \hat{u}_{h}, \hat{u}_{h}\right)=L_{\delta}\left(u_{h} ; \hat{u}_{h}\right) \\
B_{\delta}\left(u_{h}, u_{h} ; \hat{u}_{h}, \hat{u}_{h}\right)=\mu\left|u_{h}\right|_{1}^{2}+\left\|\delta^{1 / 2}\left(-\mu \Delta u_{h}+u_{h} \nabla u_{h}+\nabla p_{h}\right)\right\|_{0, h}^{2}, \\
L_{\delta}\left(u_{h} ; \hat{u}_{h}\right) \leq\left(\mu^{-1}\|f\|_{*}^{2}+\left\|\delta^{1 / 2} f\right\|_{0}^{2}\right)^{1 / 2} \\
\quad \times\left(\mu\left|u_{h}\right|_{1}^{2}+\left\|\delta^{1 / 2}\left(-\mu \Delta u_{h}+u_{h} \nabla u_{h}+\nabla p_{h}\right)\right\|_{0, h}^{2}\right)^{1 / 2} .
\end{gathered}
$$

By (3.19), (3.20), and (3.21), we have

$$
\begin{aligned}
& \left(\mu\left|u_{h}\right|_{1}^{2}+\left\|\delta^{1 / 2}\left(-\mu \Delta u_{h}+u_{h} \nabla u_{h}+\nabla p_{h}\right)\right\|_{0, h}^{2}\right)^{1 / 2} \\
& \leq\left(\mu^{-1}\|f\|_{*}^{2}+\left\|\delta^{1 / 2} f\right\|_{0}^{2}\right)^{1 / 2} .
\end{aligned}
$$

If we let $R=\mu^{-1}\left(\|f\|_{*}^{2}+\mu\left\|\delta^{1 / 2} f\right\|_{0}^{2}\right)^{1 / 2}$, then (3.22) can be rewritten as follows:

$$
\left(\left|u_{h}\right|_{1}^{2}+\mu^{-1}\left\|\delta^{1 / 2}\left(-\mu \Delta u_{h}+u_{h} \nabla u_{h}+\nabla p_{h}\right)\right\|_{0, h}^{2}\right)^{1 / 2} \leq R .
$$

In order to prove $\hat{u}_{h} \in X_{h}$ to be the unique solution of (2.5), we suppose $\hat{u}_{h}^{i}$ $(i=1,2)$ are two solutions of $(2.5)$; by the above results we easily conclude that

$$
\left(\left|u_{h}^{i}\right|_{1}^{2}+\mu^{-1}\left\|\delta^{1 / 2}\left(-\mu \Delta u_{h}^{i}+u_{h}^{i} \nabla u_{h}^{i}+\nabla p_{h}^{i}\right)\right\|_{0, h}^{2}\right)^{1 / 2} \leq R \quad(i=1,2)
$$

and

$$
\begin{aligned}
& B_{\delta}\left(u_{h}^{1}, u_{h}^{1} ; \hat{u}_{h}^{1}, \hat{w}\right)-B_{\delta}\left(u_{h}^{2}, u_{h}^{2} ; \hat{u}_{h}^{2}, \hat{w}\right) \\
& \quad=\sum_{K} \delta_{K}\left(f,\left(u_{h}^{1}-u_{h}^{2}\right) \nabla w\right)_{K} \quad \forall \hat{w} \in X_{h} .
\end{aligned}
$$


Now let $\hat{w}=\hat{u}_{h}^{1}-\hat{u}_{h}^{2}$, i.e., $\hat{w}=(w, r)=\left(u_{h}^{1}-u_{h}^{2}, p_{h}^{1}-p_{h}^{2}\right)$. We have

$$
B_{\delta}\left(u_{h}^{1}, u_{h}^{1} ; \hat{w}, \hat{w}\right)=\mu|w|_{1}^{2}+\left\|\delta^{1 / 2}\left(-\mu \Delta w+u_{h}^{1} \nabla w+\nabla r\right)\right\|_{0, h}^{2}
$$

and

$$
B_{\delta}\left(u_{h}^{1}, u_{h}^{1} ; \hat{w}, \hat{w}\right)=B_{\delta}\left(u_{h}^{1}, u_{h}^{1} ; \hat{u}_{h}^{1}, \hat{w}\right)-B_{\delta}\left(u_{h}^{1}, u_{h}^{1} ; \hat{u}_{h}^{2}, \hat{w}\right) .
$$

By using the same arguments as in the proof of Theorem 3.1.(II), based on (3.24)-(3.27), we can get an estimate similar to (3.16):

$$
\begin{aligned}
& \mu|w|_{1}^{2}+\frac{1}{2}\left\|\delta^{1 / 2}\left(-\mu \Delta w+u_{h}^{1} \nabla w+\nabla r\right)\right\|_{0, h}^{2} \\
& \quad \leq\left(N R+2 C_{0} \mu^{1 / 2} \delta_{M}^{1 / 2} h^{-\chi} R+\frac{1}{2} C_{0}^{2} \delta_{M} h^{-2 \chi} R^{2}\right)|w|_{1}^{2} .
\end{aligned}
$$

Since

$$
R=\mu^{-1}\left(\|f\|_{*}^{2}+\mu\left\|\delta^{1 / 2} f\right\|_{0}^{2}\right)^{1 / 2} \leq \mu^{-1}\|f\|_{*}+\mu^{-1 / 2} \delta_{M}^{1 / 2}\|f\|_{0},
$$

(3.28) becomes

$$
\begin{aligned}
\mu|w|_{1}^{2}\left(1-\mu^{-2} N\|f\|_{*}-\mu^{-3 / 2} \delta_{M}^{1 / 2} N\|f\|_{0}\right. \\
\left.-2 c_{0} \mu^{-1 / 2} \delta_{M}^{1 / 2} h^{-\chi} R-\frac{1}{2} C_{0}^{2} \mu^{-1} \delta_{M} h^{-2 \chi} R^{2}\right) \\
+\frac{1}{2}\left\|\delta^{1 / 2}\left(-\mu \Delta w+u_{h}^{1} \nabla w+\nabla r\right)\right\|_{0, h}^{2} \leq 0 .
\end{aligned}
$$

As $\mu^{-2} N\|f\|_{*}<1$, there exists a constant $\omega_{1} \in(0,1)$ such that $\mu^{-2} N\|f\|_{*} \leq$ $\omega_{1}<1$. Since $\delta_{M}=\alpha h^{2}, \delta_{M}^{1 / 2} h^{-\chi} \rightarrow 0$ as $h \rightarrow 0$, there is a constant $h_{0}>0$ such that for all $h \leq h_{0}$

(3.30) $\mu^{-3 / 2} \delta_{M}^{1 / 2} N\|f\|_{0}+2 C_{0} \mu^{-1 / 2} \delta_{M}^{1 / 2} h^{-\chi} R+\frac{1}{2} C_{0}^{2} \mu^{-1} \delta_{M} h^{-2 \chi} R^{2} \leq \frac{1}{2}\left(1-\omega_{1}\right)$.

By (3.29) and (3.30), we obtain

$$
\frac{1}{2}\left(1-\omega_{1}\right)\left[\mu|w|_{1}^{2}+\left\|\delta^{1 / 2}\left(-\mu \Delta w+u_{h}^{1} \nabla w+\nabla r\right)\right\|_{0, h}^{2}\right] \leq 0 .
$$

This means that $|w|_{1}^{2}=\left\|\mu^{1 / 2}\left(-\mu \Delta w+u_{h}^{1} \nabla w+\nabla r\right)\right\|_{0, h}^{2}=0$, that is, $\hat{u}_{h}^{1}=$ $\hat{u}_{h}^{2}$.

\section{Convergence of THE Method}

This section is devoted to establishing convergence results on the Galerkin/least squares finite element approximation for any choice of discrete velocity and pressure spaces.

Theorem 4.1. Assume that $f \in L^{2}(\Omega)^{n}$, and let $\left\{\hat{u}_{h}\right\}$ be a sequence of solutions of $(2.5)$ as $h$ tends to zero. Then there is a subsequence $\left\{\hat{u}_{h}\right\}$ which converges strongly to a solution $\hat{u}$ of (2.2) in the sense of

$$
\lim _{h \rightarrow 0}\left(\left|u-u_{h}\right|_{1}+\alpha^{1 / 2} h\left\|\nabla p-\nabla p_{h}\right\|_{0}\right)=0 .
$$

Proof. From Theorem 3.2 we see that $\left\{\hat{u}_{h}\right\}$ is uniformly bounded with respect to $h$, i.e., there exists a constant $C$ independent of $h$ and $\hat{u}_{h}$ such that

$$
\left(\mu\left|u_{h}\right|_{1}^{2}+\sum_{K} \delta_{K}\left\|-\mu \Delta u_{h}+u_{h} \nabla u_{h}+\nabla p_{h}\right\|_{0,2, K}^{2}\right)^{1 / 2} \leq C .
$$


Using $h / h_{K} \leq C, \delta_{K}=\alpha h_{K}^{2},(4.2)$ and an inverse inequality, we obtain

$$
\left|u_{h}\right|_{1}+\alpha^{1 / 2} h\left\|\nabla p_{h}\right\|_{0} \leq C .
$$

By (4.3) we have

$$
\left\|p_{h}\right\|_{0} \leq C(\alpha),
$$

where $C(\alpha)$ denotes a constant dependent on $\alpha$ and independent of $h$ and $\hat{u}_{h}$. Therefore, we get

$$
\left|u_{h}\right|_{1}+\left\|p_{h}\right\|_{0} \leq C(\alpha) .
$$

Consequently, by (4.5) there is a weakly converging subsequence in $V \times Q$, which for simplicity we denote again by $\left\{\hat{u}_{h}\right\}$. We will show that the weak limit $\hat{u}$ is a solution of (2.2). For this, let $I_{h}=\left(I_{h}^{1}, I_{h}^{2}\right):\left(V \cap H^{2}(\Omega)^{n}\right) \times(Q \cap$ $\left.H^{1}(\Omega)\right) \rightarrow V_{h} \times Q_{h}$ be the usual Lagrangian interpolation operator [15]. Setting $\hat{w}=I_{h} \hat{v}=\left(I_{h}^{1} v, I_{h}^{2} q\right)$ in (2.5), we conclude that, as $h$ tends to zero,

$$
\mu a(u, v)+b(u ; u, v)-(p, \operatorname{div} v)+(q, \operatorname{div} u)=(f, v)+\lim _{h \rightarrow 0} F_{h}^{1},
$$

where

$$
F_{h}^{1}=\sum_{K} \delta_{K}\left(f+\mu \Delta u_{h}-u_{h} \nabla u_{h}-\nabla p_{h},-\mu \Delta I_{h}^{1} v+u_{h} \nabla I_{h}^{1} v+\nabla I_{h}^{2} q\right)_{K} .
$$

By (4.2) and Cauchy's inequality and an inverse inequality, we have

$$
\begin{aligned}
\left|F_{h}^{1}\right| \leq & \sum_{K} \delta_{K}\|f\|_{0, K}\left\|-\mu \Delta I_{h}^{1} v+u_{h} \nabla I_{h}^{1} v+\nabla I_{h}^{2} q\right\|_{0, K} \\
& +\left(\sum_{K} \delta_{K}\left\|-\mu \Delta u_{h}+u_{h} \nabla u_{h}+\nabla p_{h}\right\|_{0, K}^{2}\right)^{1 / 2} \\
& \times\left(\sum_{K} \delta_{K}\left\|-\mu \Delta I_{h}^{1} v+u_{h} \nabla I_{h}^{1} v+\nabla I_{h}^{2} q\right\|_{0, K}^{2}\right)^{1 / 2} \\
\leq & \left(\delta_{M}\|f\|_{0}+C \delta_{M}^{1 / 2}\right)\left(\left\|-\mu \Delta I_{h}^{1} v\right\|_{0, h}+\left\|u_{h} \nabla I_{h}^{1} v+\nabla I_{h}^{2} q\right\|_{0}\right) \\
\leq & \left(\delta_{M}\|f\|_{0}+C \delta_{M}^{1 / 2}\right)\left(\left\|-\mu \Delta\left(v-I_{h}^{1} v\right)\right\|_{0, h}+\|-\mu \Delta v\|_{0}\right. \\
& \left.\quad+C_{0} h^{-\chi}\left|I_{h}^{1} v\right|_{1}+\left|q-I_{h}^{2} q\right|_{1}+|q|_{1}\right) \\
\leq & \left(C \delta_{M}^{1 / 2}+\delta_{M}\|f\|_{0}\right)\left(C\|v\|_{2}+C h^{-\chi}\|v\|_{1}+C\|q\|_{1}\right) \\
\leq & C(f, \hat{v}) \delta_{M}^{1 / 2} h^{-\chi} \leq C(f, \hat{v}) h^{1-\chi},
\end{aligned}
$$

where $C(f, \hat{v})$ denotes a constant dependent on $f$ and $\hat{v}$, but independent of $h$. This gives $\lim _{h \rightarrow 0} F_{h}^{1}=0$, since $1-\chi>0$; i.e., for $\hat{v}=(v, q) \in$ $\left(V \cap H^{2}(\Omega)^{n}\right) \times\left(Q \cap H^{1}(\Omega)\right),(4.6)$ becomes

$$
\mu a(u, v)+b(u ; u, v)-(p, \operatorname{div} v)+(q, \operatorname{div} u)=(f, v) .
$$

Since $\left(V \cap H^{2}(\Omega)^{n}\right) \times\left(Q \cap H^{1}(\Omega)\right)$ is dense in $V \times Q$, we obtain that

$$
\begin{gathered}
\mu a(u, v)+b(u ; u, v)-(p, \operatorname{div} v)+(q, \operatorname{div} u) \\
\quad=(f, v) \quad \forall \hat{v}=(v, q) \in V \times Q ;
\end{gathered}
$$

i.e., $(u, p)$ is a weak solution. 
Now we prove that $\lim _{h \rightarrow 0}\left(\left|u-u_{h}\right|_{1}+\alpha^{1 / 2} h\left|p-p_{h}\right|_{1}\right)=0$. In fact, for $f \in L^{2}(\Omega)^{n}$, the solution $\hat{u}=(u, p)$ of $(2.2)$ belongs to $\left(H_{0}^{1}(\Omega) \cap H^{2}(\Omega)\right)^{n} \times$ $\left(H^{1}(\Omega) / R\right)$ and there holds $\mu a(u, u)=(f, u)$. Therefore,

$$
\begin{aligned}
\mu \mid u_{h}- & \left.I_{h}^{1} u\right|_{1} ^{2}+\left\|\delta^{1 / 2} \nabla\left(p_{h}-I_{h}^{2} p\right)\right\|_{0, h}^{2} \\
= & \mu a\left(u_{h}-I_{h}^{1} u, u_{h}-I_{h}^{1} u\right)+\sum_{K} \delta_{K}\left(\nabla\left(p_{h}-I_{h}^{2} p\right), \nabla\left(p_{h}-I_{h}^{2} p\right)\right)_{K} \\
= & \mu a\left(u_{h}, u_{h}\right)+\sum_{K} \delta_{K}\left(\nabla p_{h}, \nabla p_{h}\right)-\mu a\left(2 u_{h}-I_{h}^{1} u, I_{h}^{1} u\right) \\
& -\sum_{K} \delta_{K}\left(\nabla\left(2 p_{h}-I_{h}^{2} p\right), \nabla I_{h}^{2} p\right)_{K} .
\end{aligned}
$$

Recalling that $\hat{u}_{h}=\left(u_{h}, p_{h}\right)$ satisfies $(2.5)$, we have

$$
\mu a\left(u_{h}, u_{h}\right)=\left(f, u_{h}\right)+F_{h}^{2},
$$

where

$$
F_{h}^{2}=\sum_{K} \delta_{K}\left(f+\mu \Delta u_{h}-u_{h} \nabla u_{h}-\nabla p_{h},-\mu \Delta u_{h}+u_{h} \nabla u_{h}+\nabla p_{h}\right)_{K}
$$

and

$$
\begin{aligned}
F_{h}^{2}= & -\sum_{K} \delta_{K}\left(\nabla p_{h}, \nabla p_{h}\right)+\sum_{K} \delta_{K}\left(f+\mu \Delta u_{h}-u_{h} \nabla u_{h}, \nabla p_{h}\right)_{K} \\
& +\sum_{K} \delta_{K}\left(f+\mu \Delta u_{h}-u_{h} \nabla u_{h}-\nabla p_{h},-\mu \Delta u_{h}+u_{h} \nabla u_{h}\right)_{K} .
\end{aligned}
$$

By (4.10), (4.11), and (4.12), we get

$$
\begin{aligned}
\mu \mid u_{h}- & \left.I_{h}^{1} u\right|_{1} ^{2}+\left\|\delta^{1 / 2} \nabla\left(p_{h}-I_{h}^{2} p\right)\right\|_{0, h}^{2} \\
= & \left(f, u_{h}\right)-\mu a\left(2 u_{h}-I_{h}^{1} u, I_{h}^{1} u\right)-\sum_{K} \delta_{K}\left(\nabla\left(2 p_{h}-I_{h}^{2} p\right), \nabla I_{h}^{2} p\right)_{K} \\
& +\sum_{K} \delta_{K}\left(f+\mu \Delta u_{h}-u_{h} \nabla u_{h}, \nabla p_{h}\right)_{K} \\
& +\sum_{K} \delta_{K}\left(f+\mu \Delta u_{h}-u_{h} \nabla u_{h}-\nabla p_{h},-\mu \Delta u_{h}+u_{h} \nabla u_{h}\right)_{K} \\
= & :\left(f, u_{h}\right)-\mu a\left(2 u_{h}-I_{h}^{1} u, I_{h}^{1} u\right)+F_{h}^{3} .
\end{aligned}
$$

By using the same techniques used in deriving the estimates of $F_{h}^{1}$, we obtain

$$
\left|F_{h}^{3}\right| \leq C \delta_{M}^{1 / 2} h^{-\chi} .
$$

Thus, we conclude from (4.13) and (4.14) that as $h \rightarrow 0$

$$
\lim _{h \rightarrow 0}\left(\mu\left|u_{h}-I_{h}^{1} u\right|_{1}^{2}+\left\|\delta^{1 / 2} \nabla\left(p_{h}-I_{h}^{2} p\right)\right\|_{0}^{2}\right)=(f, u)-\mu a(u, u)=0 .
$$

By virtue of the definition of $\delta$ and the assumption $h / h_{K} \leq C,(4.15)$ yields

$$
\lim _{h \rightarrow 0}\left(\left|u_{h}-I_{h}^{1} u\right|_{1}+\alpha^{1 / 2} h\left|p_{h}-I_{h}^{2} p\right|_{1}\right)=0 .
$$

Finally, $\lim _{h \rightarrow 0}\left(\left|u-u_{h}\right|_{1}+\alpha^{1 / 2} h\left|p-p_{h}\right|_{1}\right)=0$. 
Remark 2. In the proof of (4.14), we used (4.2) and $\left\|u_{h}\right\|_{0, \infty} \leq C_{0} h^{-\chi}\left|u_{h}\right|_{1}$ and $\left(\sum_{K}\left\|\Delta u_{h}\right\|_{0, K}^{2}\right)^{1 / 2} \leq C h^{-1}\left|u_{h}\right|_{1}$.

Theorem 4.2. Assume that $\mu^{-2} N\|f\|_{*}<1$ and that the exact solution $\hat{u}=(u, p)$ of (2.2) belongs to the space $\left(W_{0}^{1, \infty}(\Omega) \cap W^{l+1}(\Omega)\right)^{n} \times W^{k+1}(\Omega), l, k \in \mathbb{N}$. Then there is a positive constant $h^{*}$ such that the following error estimate holds for the solution $\hat{u}_{h}=\left(u_{h}, p_{h}\right)$ of (2.5) for $h \leq h^{*}$ :

$$
\begin{aligned}
& \left(\mu\left|u-u_{h}\right|_{1}^{2}+\left\|\delta^{1 / 2}\left[-\mu \Delta\left(u-u_{h}\right)+u_{h} \nabla\left(u-u_{h}\right)+\nabla\left(p-p_{h}\right)\right]\right\|_{0, h}^{2}\right)^{1 / 2} \\
& \quad \leq C\left(h^{l}+h^{k+1}\right),
\end{aligned}
$$

where the constant $C$ depends on the seminorms $|u|_{1, \infty},|u|_{l+1},|p|_{k+1}$ of the exact solution of (2.2).

Proof. According to Theorem 2.1 and Theorem 3.2, both problems (2.2) and (2.5) have uniquely determined solutions. Let $\hat{w}_{h}=I_{h} \hat{u}-\hat{u}_{h}$, i.e., $\hat{w}_{h}=$ $\left(w_{h}, r_{h}\right)=\left(I_{h}^{1} u-u_{h}, I_{h}^{2} p-p_{h}\right)$. It is easy to see that

(4.16) $S_{1}=: B_{\delta}\left(u_{h}, u_{h} ; \hat{w}_{h}, \hat{w}_{h}\right)=\mu\left|w_{h}\right|_{1}^{2}+\left\|\delta^{1 / 2}\left(-\mu \Delta w_{h}+u_{h} \nabla w_{h}+\nabla r_{h}\right)\right\|_{0, h}^{2}$,

and from (2.5), (2.6) we derive that

$$
\begin{aligned}
S_{1} & =B_{\delta}\left(u_{h}, u_{h} ; I_{h} \hat{u}, \hat{w}_{h}\right)-B_{\delta}\left(u_{h}, u_{h} ; \hat{u}_{h}, \hat{w}_{h}\right) \\
& =B_{\delta}\left(u_{h}, u_{h} ; I_{h} \hat{u}, \hat{w}_{h}\right)-B_{\delta}\left(u, u_{h} ; \hat{u}, \hat{w}_{h}\right) ;
\end{aligned}
$$

i.e.,

$$
S_{1}=S_{2}+S_{3}+S_{4}
$$

where

$$
\begin{aligned}
& S_{2}=\mu a\left(I_{h}^{1} u-u, w_{h}\right)-\left(I_{h}^{2} p-p, \operatorname{div} w_{h}\right), \\
& S_{3}=\sum_{K} \delta_{K}\left(-\mu \Delta\left(I_{h}^{1} u-u\right)+u_{h} \nabla I_{h}^{1} u-u \nabla u+\nabla\left(I_{h}^{2} p-p\right),-\mu \Delta w_{h}+u_{h} \nabla w_{h}+\nabla r_{h}\right)_{K}, \\
& S_{4}=b\left(u_{h} ; I_{h}^{1} u, w_{h}\right)-b\left(u ; u, w_{h}\right)+\left(r_{h}, \operatorname{div}\left(I_{h}^{1} u-u\right)\right) .
\end{aligned}
$$

By using well-known interpolation error estimates [15], it is easy to get

$$
\begin{gathered}
\left|S_{2}\right| \leq C\left(h^{l}+h^{k+1}\right)\left|w_{h}\right|_{1}, \\
\left|S_{3}\right| \leq \frac{1}{4} \sum_{K} \delta_{K}\left\|-\mu \Delta w_{h}+u_{h} \nabla w_{h}+\nabla r_{h}\right\|_{0, K}^{2}+C \delta_{M}\left(h^{2 l-2}+h^{2 k}\right) \\
+C \delta_{M}\left\|u_{h} \nabla\left(I_{h}^{1} u-u\right)+\left(u_{h}-u\right) \nabla u\right\|_{0}^{2} \\
\leq \frac{1}{4}\left\|\delta^{1 / 2}\left(-\mu \Delta w_{h}+u_{h} \nabla w_{h}+\nabla r_{h}\right)\right\|_{0, h}^{2} \\
+C \delta_{M}\left|w_{h}\right|_{1}^{2}+C \delta_{M}\left(h^{2 l-2}+h^{2 k}+h^{2 l-2 \chi}+h^{2 l+2}\right) .
\end{gathered}
$$

It should be mentioned that the constants $C$ in (4.18) and (4.19) depend on the seminorms of the exact solution.

For $S_{4}$, by using Green's formula, we have

$$
\left(r_{h}, \operatorname{div}\left(I_{h}^{1} u-u\right)\right)=\left(u_{h} \nabla w_{h}, I_{h}^{1} u-u\right)-\left(u_{h} \nabla w_{h}+\nabla r_{h}, I_{h}^{1} u-u\right),
$$


and then

$$
\begin{aligned}
S_{4}= & b\left(u_{h} ; I_{h}^{1} u-u, w_{h}\right)+b\left(I_{h}^{1} u-u ; u, w_{h}\right)-b\left(w_{h} ; u, w_{h}\right) \\
& +\left(u_{h} \nabla w_{h}, I_{h}^{1} u-u\right)-\left(u_{h} \nabla w_{h}+\nabla r_{h}, I_{h}^{1} u-u\right) .
\end{aligned}
$$

Recalling that $\hat{u}_{h}$ is bounded and that for the exact solution $\hat{u}$ we have $|u|_{1} \leq$ $\mu^{-1}\|f\|_{*}$, and using an inverse estimate, we get

$$
\begin{aligned}
\left|S_{4}\right| \leq & C h^{l}\left|w_{h}\right|_{1}+\mu^{-1} N\|f\|_{*}\left|w_{h}\right|_{1}^{2} \\
& +\left|\sum_{K}\left(-\mu \Delta w_{h}+u_{h} \nabla w_{h}+\nabla r_{h}, I_{h}^{1} u-u\right)_{K}\right| \\
& +\left|\sum_{K}\left(-\mu \Delta w_{h}, I_{h}^{1} u-u\right)_{K}\right| \\
\leq & C h^{l}\left|w_{h}\right|_{1}+\mu^{-1} N\|f\|_{*}\left|w_{h}\right|_{1}^{2}+C \delta_{\min }^{-1} h^{2 l+2} \\
& +\frac{1}{4}\left\|\delta^{1 / 2}\left(-\mu \Delta w_{h}+u_{h} \nabla w_{h}+\nabla r_{h}\right)\right\|_{0, h}^{2},
\end{aligned}
$$

where $\delta_{\min }=\min _{x \in \Omega} \delta=\inf _{K} \delta_{K}$. Combining (4.16) with (4.17)-(4.20), we have

$$
\begin{array}{r}
\mu|w|_{1}^{2}\left(1-\mu^{-2} N\|f\|_{*}-C \delta_{M}\right)+\frac{1}{2}\left\|\delta^{1 / 2}\left(-\mu \Delta w_{h}+u_{h} \nabla w_{h}+\nabla r_{h}\right)\right\|_{0, h}^{2} \\
\leq C\left(h^{l}+h^{k+1}\right)\left|w_{h}\right|_{1}+C \delta_{M}\left(h^{2 l-2}+h^{2 k}+h^{2 l-2 \chi}\right)+C \delta_{\min }^{-1} h^{2 l+2} .
\end{array}
$$

Taking into account $\delta_{M}=\alpha h^{2}$ and $\mu^{-2} N\|f\|_{*}<1$, we may conclude that there exists a sufficiently small $h^{*}>0$ such that

$$
\begin{aligned}
& \left(\mu\left|w_{h}\right|_{1}^{2}+\left\|\delta^{1 / 2}\left(-\mu \Delta w_{h}+u_{h} \nabla w_{h}+\nabla r_{h}\right)\right\|_{0, h}^{2}\right)^{1 / 2} \\
& \leq C\left(h^{l}+h^{k+1}+\delta_{\min }^{-1 / 2} h^{l+1}\right)
\end{aligned}
$$

for all $h \leq h^{*}$. Since $\delta_{K}=\alpha h_{K}^{2}, h / h_{K} \leq C$, we then obtain

$$
\begin{aligned}
& \left(\mu\left|w_{h}\right|_{1}^{2}+\left\|\delta^{1 / 2}\left(-\mu \Delta w_{h}+u_{h} \nabla w_{h}+\nabla r_{h}\right)\right\|_{0, h}^{2}\right)^{1 / 2} \\
& \quad \leq C\left(h^{l}+h^{k+1}\right) .
\end{aligned}
$$

Noting that $\hat{w}_{h}=I_{h} \hat{u}-\hat{u}_{h}$, and using the triangle inequality, we finally get

$$
\begin{aligned}
& \left(\mu\left|u-u_{h}\right|_{1}^{2}+\left\|\delta^{1 / 2}\left[-\mu \Delta\left(u-u_{h}\right)+u_{h} \nabla\left(u-u_{h}\right)+\nabla\left(p-p_{h}\right)\right]\right\|_{0, h}^{2}\right)^{1 / 2} \\
& \leq C\left(h^{l}+h^{k+1}\right) .
\end{aligned}
$$

Remark 3. By using Nitsche's duality technique, we can also get $L^{2}$-error estimates for velocity and pressure.

Remark 4. If the finite element pressure subspace $Q$ belongs only to $L_{0}^{2}(\Omega)$, we need to add the boundary integral term $\sum_{K} \beta h_{K} \oint_{\partial K}[q][r] d s$ to $B_{\delta}\left(u, u_{h} ; \hat{v}, \hat{w}\right)$ (where $\beta>0,[q]=q_{+}-q_{-}$) in order to obtain corresponding convergence results.

\section{Conclusion}

A finite element method of Galerkin/least squares-type for approximating the stationary N-S equations in primitive variables is presented with the following characteristics: 
(i) The method exhibits stable and convergent approximation with optimal rate for any choice of the discrete velocity and pressure spaces, in contrast with the Galerkin mixed methods, in which the discrete B-B condition is required. For the 3-dimensional analysis, this point has important significance because of the implementational simplicity of lower and equal-order interpolations.

(ii) The method is variationally consistent, and the parameter $\alpha>0$ can be arbitrarily chosen, yielding practical convenience and improved convergence error estimates compared to the associated penalty-type method [12].

\section{ACKNOWLEDGMENT}

The authors would like to thank the referee for helpful comments and suggestions.

\section{BIBLIOGRAPHY}

1. V. Girault and P. A. Raviart, Finite element methods for Navier-Stokes equations, Lecture Notes in Math., vol. 749, Springer-Verlag, Berlin and New York, 1981.

2. T. J. R. Hughes, L. P. Franca, and M. Balestra, A new finite element formulation for computational fluid dynamics. V. Circumventing the Babuška-Brezzi condition: A stable PetrovGalerkin formulation of the Stokes problem accommodating equal-order interpolation, Comput. Methods Appl. Mech. Engrg. 59 (1986), 85-99.

3. T. J. R. Hughes and L. P. Franca, A new finite element formulation for computational fluid dynamics. VII. The Stokes problem with various well posed boundary conditions, symmetric formulations that converge for all velocity/pressure spaces. Comput. Methods Appl. Mech. Engrg. 65 (1987), 85-96.

4. F. Brezzi and J. Douglas, Jr., Stabilized mixed methods for the Stokes problem, Numer. Math. 53 (1988), 225-235.

5. J. Douglas, Jr. and J. P. Wang, An absolutely stabilized finite element method for the Stokes problem, Math. Comp. 52 (1989), 495-508.

6. L. P. Franca and T. J. Hughes, Two classes of mixed finite element methods, Comput. Methods Appl. Mech. Engrg. 69 (1989), 89-129.

7. T. J. Hughes and T. E. Tezduyar, Finite element methods for first-order hyperbolic systems with particular emphasis on the compressible Euler equations, Comput. Methods Appl. Mech. Engrg. 45 (1984), 217-284.

8. C. Johnson and J. Saranen, Streamline diffusion methods for the incompressible Euler and Navier-Stokes equations, Math. Comp. 47 (1986), 1-18.

9. P. Hansbo and A. Szepessy, A velocity-pressure streamline diffusion finite element method for the incompressible Navier-Stokes equations, Comput. Methods Appl. Mech. Engrg. 84 (1990), 175-192.

10. Tian-Xiao Zhou, Stabilized Galerkin finite element methods based on homology family of variational principles. II. Application to the Navier-Stokes equations, Math. Numer. Sinica (to appear).

11. G. Lube and L. Tobiska, A nonconforming finite element method of streamline diffusion type for the incompressible Navier-Stokes equations, J. Comput. Math. 8 (1990), 147-158.

12. L. Tobiska and G. Lube, A modified streamline diffusion method for solving the stationary $N$-S equations, Preprint.

13. Tian-Xiao Zhou, Min-Fu Feng, and Hua-Xing Xiong, A new approach to stability of finite elements under divergence constraints, J. Comput. Math. 10 (1992), 1-15.

14. T. J. R. Hughes, L. P. Franca, and G. M. Hulbert, A new finite element formulation for computational fluid dynamcis. VIII. The Galerkin/least-squares method for advective-diffusive equations, Comput. Methods Appl. Mech. Engrg. 89 (1989), 173-189. 
15. P. G. Ciarlet, The finite element method for elliptic problems, North-Holland, Amsterdam, 1978.

16. L. P. Franca, S. L. Frey, and T. J. R. Hughes, Stabilized finite element methods. I. Application to the advective-diffusion model, Preprint, Sept. 1990.

Computing Technology Research Institute, Chinese Aeronautical Establishment, XI'AN, China 710068

Department of Mathematics, Xi'an Jiaotong University, Xi'an, China 